\title{
Electrical Conduction in HVDC Mass-impregnated Paper Cable
}

\author{
M. J. P. Jeroense \\ NKF Kabel BV, Delft \\ and F. H. Kreuger \\ High Voltage Laboratory, \\ Delft University of Technology, Delft, \\ The Netherlands
}

\begin{abstract}
HVDC cables play an important role in a growing number of HVDC links. For almost all of these cables, mass impregnated paper is used as electrical insulation. The electrical conductivity of this insulation is given by a commonly used empirical formula. This formula takes into account the temperature and stress dependency of the conductivity. This paper derives a physics-based equation describing ionic conduction. In good approximation this theoretical formula gives the same results as the empirical one. This makes it most likely that the conduction in paper mass impregnated cables is of the ionic kind. The derived formula uses some physical parameters like the inter-potential well distance, the potential well depth and the carrier concentration. Numerical values of these quantities for mass impregnated paper are given. According to the proposed model, the stress dependency results from the inter-potential well distance only. The temperature dependency results merely from the depth of the potential energy wells. In addition some possible underlying physical processes are described.
\end{abstract}

\section{INTRODUCTION}

F ROM the early beginning of the development of the H HVDC cable, these cables haven been insulated with mass impregnated paper. The electric field in the cable insulation depends on the conduction properties of the insulating material and the geometry of the cable. Although the geometry is exactly known, the conduction properties of the impregnated material are uncertain.

In recent years worldwide measurements resulted in empirical formulas, describing the conductivity of mass impregnated paper as a function of temperature and field strength [1-8]. All these formulas represent the measured conductivity data with a reasonable accuracy. The most commonly used empirical formula is

$$
\sigma=\sigma_{0} \exp [\alpha T+\gamma E]
$$

where $\sigma$ stands for electrical conduction, $\sigma_{0}$ for the conduction at zero temperature and zero electrical field, $\alpha$ for the temperature dependency coefficient, $\gamma$ for the field dependency coefficient, $T$ for the temperature and $E$ for the electrical field. Although it is assumed that the conduction mechanism of mass impregnated paper is of the ionic kind $[6,15]$, this empirical formula lacks a physical basis. In this paper it will be shown that it is possible to connect a physically based conduction formula to the empirical one and to describe the quantitative aspects of the underlying conduction process. Some possible con- 
duction mechanisms are discussed.

\section{THEORY}

Let us assume a medium in which ionic conduction takes place. The medium consists of $n_{1}$ particles of type 1, $n_{2}$ particles of type 2 and in general $n_{i}$ particles of type $i$ per unit volume. Assign a charge $q_{i}$ to each particle, where $q_{i}=w_{i} e, w_{i}$ is an integer (zero, positive or negative) and $e$ denotes the charge of one electron. The total number of particles per unit volume is given by

$$
N_{0}=\sum_{i} n_{i}
$$

In this medium a charged particle can be trapped by a host particle, usually a larger polymer molecule. Physically this means that the particle is trapped in a potential energy well of height $H$. In fact, all host particles together form a 3-dimensional potential energy landscape through which the particle travels.

Particles of different kinds can be caught by different types of host particles, thus taking into account different conduction processes at a time. This means that particles of different kinds experience different potential energy landscapes. For this reason the height of the potential energy for a particle of type $i$ will be denoted by $H_{i}$. The probability per unit time (from now on the addition 'per unit time' will be omitted) that a particle of type $i$ escapes from an equilibrium position depends on the temperature $T$, the depth of the potential energy well $H_{i}$, the attempt-to-escape frequency $\omega_{i}$ and can be found to be

$$
p_{i}=\frac{\omega_{i}}{2 \pi} \exp \left[-\frac{H_{i}}{k T}\right]
$$

where $k$ is the Boltzmann constant $[10,11,19]$.

After having escaped, the particle falls into the potential energy well of the next host. In the following the assumption is made that no particle will cross two potential energy wells at a time. This is justified, because $p_{i}^{2} \ll p_{i}$.

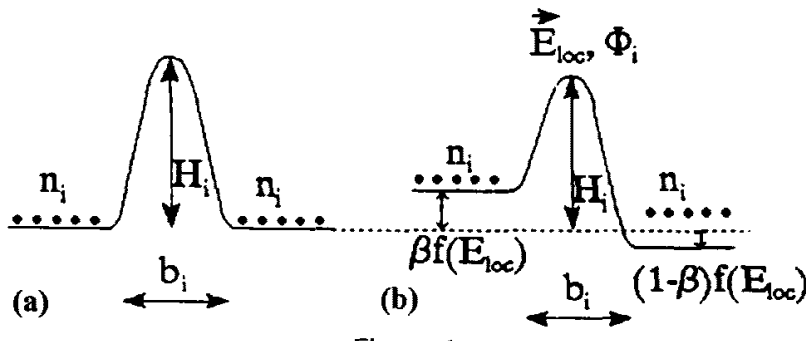

Figure 1.

(a) The situation around a potential energy well in absence of an electric field. (b) The situation around a potential energy well with an electric field.
Let us concentrate on what will happen around one potential energy well. In the absence of an electric field the probability $p_{i}^{1,2}$ that a particle of type $i$ will cross the well from left to right is the same as the probability $p_{i}^{2,1}$ that a particle of type $i$ will cross the well from right to left, and equals $p_{i}$. Thus the net particle flux across the well is zero (Figure 1(a)).

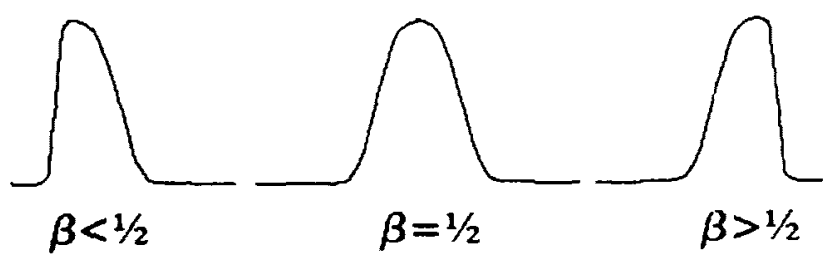

Figure 2.

The effect of the symmetry factor on the shape of the potential energy well.

When an electric field is applied over the well, the probability $p_{i}^{1,2}$ that a particle of type $i$ will cross the well in the direction of the field is given by

$$
p_{i}^{1,2}=\frac{\omega_{i}}{2 \pi} \exp \left[\frac{(1-\beta) q_{i} b_{i} E_{l o c}-H_{i}}{k T}\right]
$$

where $E_{l o c}$ denotes the microscopic electric field in the region of the potential energy well caused by the macroscopic electric field $E, b_{i}$ denotes the distance between two equilibrium positions for a particle of type $i$, and $\beta$ is a symmetry factor $[10,11,16,19]$. The probability per unit time $p_{i}^{2,1}$ of a particle of type $i$ to cross the well against the direction of the field is given by

$$
p_{i}^{2,1}=\frac{\omega_{i}}{2 \pi} \exp \left[\frac{-\beta q_{i} b_{i} E_{l o c}-H_{i}}{k T}\right]
$$

The symmetry factor $\beta$ describes the shape of the potential energy well (Figure 2) [16]. A high degree of symmetry $(\beta=1 / 2)$ results in an equal field induced change in probability for a particle to cross the well in both directions. A high degree of asymmetry $(\beta=0$ or 1$)$ results in an unequal field induced change in probability for a particle to cross the well from left to right in comparison with a jump from right to left (see Figure 1(b)). Now the net particle flux of type $i$ across the well in the direction of the field is given by

$$
\begin{aligned}
\Phi_{i}= & n_{i}\left(p_{i}^{1,2}-p_{i}^{2,1}\right)=n_{i} \frac{\omega_{i}}{2 \pi} \exp \left[-\frac{H_{i}}{k T}\right] \times \\
& \left\{\exp \left[\frac{(1-\beta) q_{i} b_{i} E_{l o c}}{k T}\right]-\exp \left[\frac{-\beta q_{i} b_{i} E_{l o c}}{k T}\right]\right\}
\end{aligned}
$$

If the particles have no charge they do not contribute to the conduction process. However if particles of type $i$ 
carry a charge $q_{i}$, they do contribute to the conduction process according to

$$
j_{i}=q_{i} \Phi_{i}
$$

where $j_{i}$ denotes the current density contribution of particles of type $i$. The conductivity in the region of the energy well caused by particles of type $i$ can now be computed by

$$
\sigma_{i, l o c}=\frac{j_{i}}{E_{l o c}}
$$

The total conductivity of the medium in the region of the energy well can be found by considering that every kind of particle and therefore every conduction process contributes simultaneously to the conduction. The total conductivity, according to this model is therefore given by

$$
\begin{aligned}
\sigma_{l o c} & =\sum_{i} \sigma_{i, l o c} \\
& =\frac{1}{E_{l o c}} \times \sum_{i} q_{i} n_{i} \frac{\omega_{i}}{2 \pi} \exp \left[-\frac{H_{i}}{k T}\right] \times \\
& \left\{\exp \left[\frac{(1-\beta) q_{i} b_{i} E_{l o c}}{k T}\right]-\exp \left[\frac{-\beta q_{i} b_{i} E_{l o c}}{k T}\right]\right\}
\end{aligned}
$$

Two simplifications can be made. At first, if we are only interested in the conductivity of the medium on a macroscopic scale, we can state that

$$
E_{l o c}=E
$$

Secondly, because we do not know the different conduction processes which take place, we consider the sum of all conduction processes to be the net result of one virtual conduction process only,

$$
\begin{aligned}
\sum_{i} n_{i} & =N_{0} \\
\sum_{i} \omega_{i} & =\omega_{0} \\
\sum_{i} q_{i} & =q \\
\sum_{i} H_{i} & =H
\end{aligned}
$$

These two simplifications lead to the modified conduction formula

$$
\begin{aligned}
\sigma= & \frac{N_{0} q \omega}{2 \pi E} \exp \left[-\frac{H}{k T}\right] \times \\
& \left\{\exp \left[\frac{(1-\beta) q b E}{k T}\right]-\exp \left[\frac{-\beta q b E}{k T}\right]\right\}
\end{aligned}
$$

In the symmetric case, $\beta=1 / 2$, the formula reduces in

$$
\sigma=\frac{N_{0} q \omega_{0}}{\pi E} \exp \left[-\frac{H}{k T}\right] \sinh \left[\frac{q b E}{2 k T}\right]
$$

whereas for the asymmetric case, $\beta=1$, we find

$$
\sigma=\frac{N_{0} q \omega_{0}}{2 \pi E} \exp \left[-\frac{H}{k T}\right]\left\{1-\exp \left[\frac{-q b E}{k T}\right]\right\}
$$

and for the asymmetric case, $\beta=0$, we end up with

$$
\sigma=\frac{N_{0} q \omega_{0}}{2 \pi E} \exp \left[-\frac{H}{k T}\right]\left\{\exp \left[\frac{q b E}{k T}\right]-1\right\}
$$

\section{RESULTS AND DISCUSSION}

The conductivity has been computed for three types of insulation (Table 1) with the empirical (1) and the theoretical Equations (13), (14) and (15). The three insulation types are described in the caption of Table 1. All mentioned empirical and theoretical equations use constants. The empirical one uses $\sigma_{0}, \alpha, \beta$ and the theoretical ones use $N_{0}, b, q, \omega_{0}$ and $H$. A mean charge $q$ of one electron charge $e$ is assumed as a representative charge for the whole conduction process. The attemptto-escape frequency $\omega_{0}$ is hard to measure directly, but varies from $10^{10}$ to $10^{14}$ according to $[10,11]$. Here a value of $10^{12}$ is taken. For the remaining constants, data from Table 1 have been used. These data have been found by an iterative process using the asymmetric conduction Equation (15).

Table 1.

The table gives values of the constants of the empirical (1) and theoretical formula for which they match over a range that reflect the actual working conditions. The theoretical formula (15) with symmetry factor $=0$ has been used here. A value of $10^{12}$ is taken for $\omega_{0}$. A mean charge $q$ of one electron charge $e$ is assumed. The numbers for $H$ apply to room temperature $\left(20^{\circ} \mathrm{C}\right)$. Insulation type 1 represents the properties of mass impregnated paper used for actual HVDC cables $[1,2$, $3,4,5,6,7,8]$. Insulation type 2 represents the properties of non-impregnated or gas-filled preimpregnated paper [6], whereas insulation type 3 is a hypothetic one.

\begin{tabular}{|c|c|c|c|c|c|c|}
\hline \multirow{3}{*}{$\begin{array}{c}\text { Insulation } \\
\text { type }\end{array}$} & \multicolumn{3}{|c|}{ Empirical } & \multicolumn{3}{c|}{ Theoretical } \\
\cline { 2 - 7 } & $\begin{array}{c}\sigma_{0}^{-1} \mathrm{~m}^{-1} \\
\Omega^{-1}\end{array}$ & $\begin{array}{c}\alpha \\
\mathrm{C}^{-1}\end{array}$ & $\begin{array}{c}\gamma \\
\mathrm{mm} / \mathrm{kV}\end{array}$ & $\begin{array}{c}N \\
\mathrm{~m}^{-3}\end{array}$ & $\begin{array}{c}H \\
\mathrm{eV}\end{array}$ & $\begin{array}{c}b \\
\mathrm{~nm}\end{array}$ \\
\hline 1 & $1 \times 10^{-16}$ & 0.1 & 0.03 & $1.7 \times 10^{15}$ & 0.9 & 1.3 \\
2 & $1 \times 10^{-16}$ & 0.1 & 0.08 & $1.7 \times 10^{15}$ & 0.9 & 2.7 \\
3 & $1 \times 10^{-16}$ & 0.05 & 0.03 & $6.1 \times 10^{15}$ & 0.5 & 1.3 \\
\hline
\end{tabular}

Figure 3 shows the conductivity data computed for insulation types 1 and 2. Comparison of the curves, computed with both the theoretical (15) and the empirical formula (1), shows an astonishing similarity. The values for temperature and stress reflect actual service conditions. The maximum deviation of the theoretical Equation (15) from the empirical one is never $>60 \%$ 

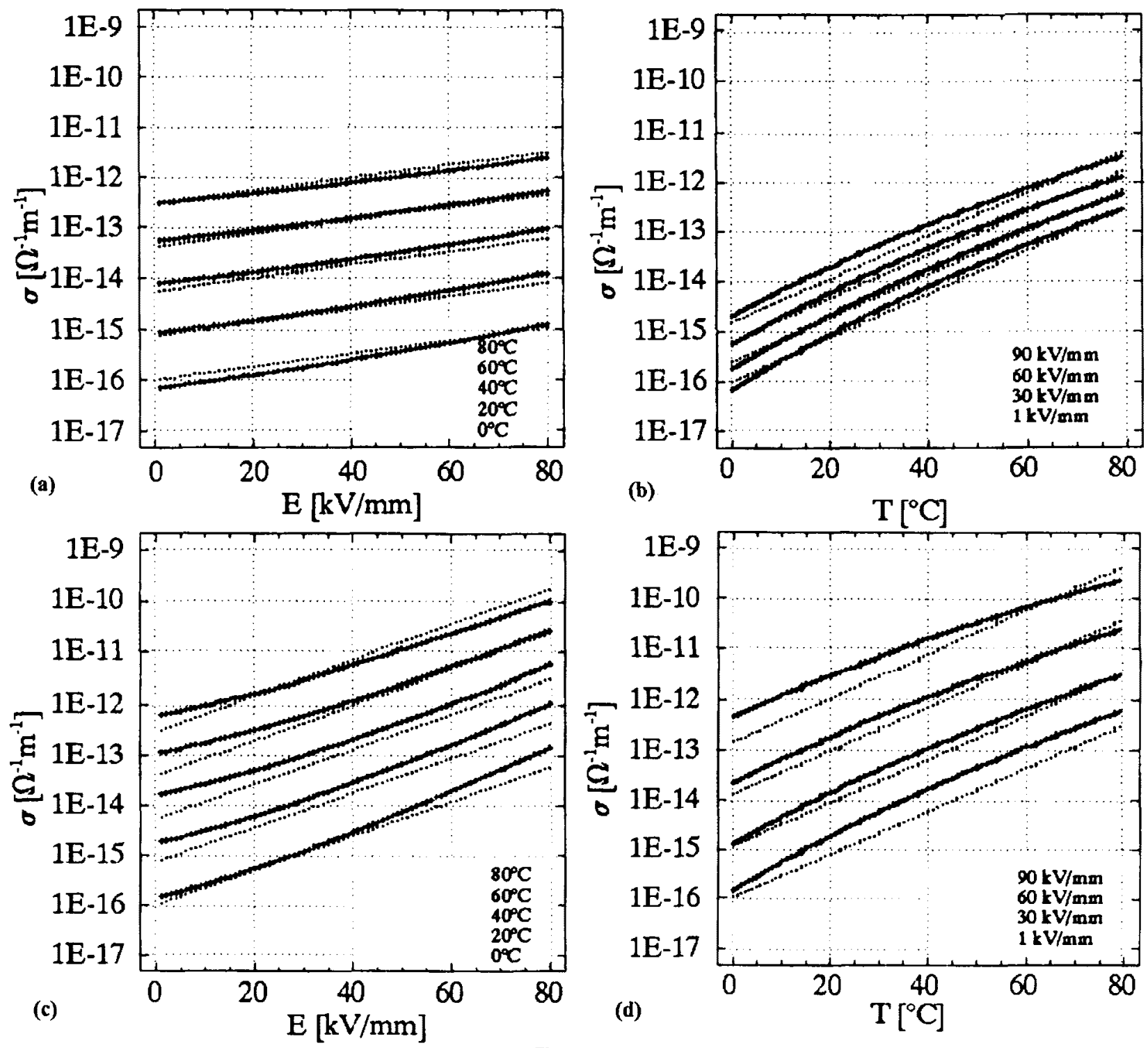

Figure 3.

(a) Comparison between empirical and theoretical curves. The Figure shows the stress dependency at different temperatures $\left(80^{\circ} \mathrm{C}\right.$ for the upper curve, $0^{\circ} \mathrm{C}$ for the lower one). Here data from Table 1 insulation type 1 are taken. (b) Temperature dependency at different stresses $(90 \mathrm{kV} / \mathrm{mm}$ for the upper curve, $1 \mathrm{kV} / \mathrm{mm}$ for the lower one). Here data from Table 1 insulation type 1 are taken. (c) Stress dependency at different temperatures $\left(80^{\circ} \mathrm{C}\right.$ for the upper curve, $0^{\circ} \mathrm{C}$ for the lower one). Here data from Table 1 insulation type 2 are taken. (d) Temperature dependency at different stresses. Here data from Table 1 insulation type 2 are taken.

in the whole range of 7 decades. The similarity of data is reached while varying in two dimensions (field $E$ and temperature $T$ ) and by using just one set of values for the parameters $N_{0}, b$ and $H$. This similarity in data makes it most likely that the electrical conduction in mass impregnated paper is of the ionic kind. The numbers for $N_{0}, b$ and $H$ (Table 1 ) are in good agreement with those found by other authors [12]. Comparison of the empirical Equation (1) with the symmetric conduc- tion Equation (13) roughly requires a doubling of the mean distance between two equilibrium points $b$ to arrive at similarity of data. However, using (13) ends up with a higher deviation in the low field region $(<15 \mathrm{kV} / \mathrm{mm})$. Still the deviation is never $>60 \%$ over the whole range of 7 decades.

Comparison of the empirical Equation (1) with the asymmetric conduction Equation (14) gives no reasonable match of empirical and theoretical data. The same 
procedure has been followed using other theoretical formulas of well known conduction processes as the PooleFrenkel model, the modified Poole-Frenkel model and Schottky emission [14]. None of these formulas, however, resulted in the similarity using just one set of parameters while varying field $E$ and temperature $T$.

The effect of using another value of the attempt-toescape frequency $\omega_{0}$ or the charge $q$ is directly reflected in the parameter $N_{0}$. For instance, a doubling of $\omega_{0}$ requires a halving of $N_{0}$.

If the empirical constant $\alpha$ is changed, only the theoretical constants $H$ and $N$ have to be changed to arrive at the same similarity of the results (compare insulation types 1 and 3 from Table 1). However, if empirical constant $\gamma$ is changed, only the theoretical constant $b$ has to be changed to obtain similarity (compare insulation types 1 and 2 from Table 1). It can therefore be concluded that, according to the conduction model here proposed, stress dependency is ruled by the mean distance between two equilibrium points, whereas the temperature dependency is governed by the mean depth of the potential energy well. The higher the stress dependency, the larger the distance between two equilibrium points and the higher the temperature dependency, the higher the potential energy well.

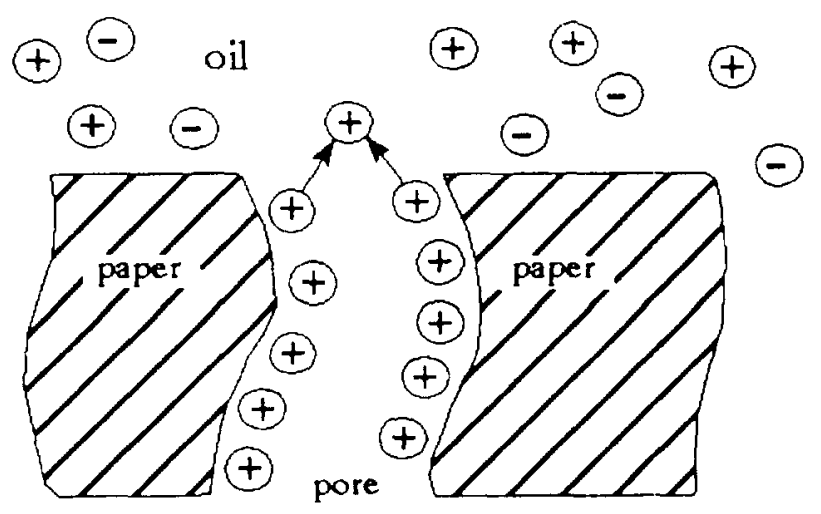

Figure 4.

Traveling ions through a paper pore.

Various authors mention different conduction mechanisms that may be responsible for ionic conduction. In the following a selection is made from these theories which are supposed to be valid for industrial mass impregnated paper. The impregnated paper will be treated here as a homogeneous insulation and not as a composite.

1. There are many examples of contamination which take place in an industrial insulation such as mass impregnated paper. To mention a few: dissolution of its own oxidation products, dissolution of metal, dissolution of additives like antioxidant, inhibitor, antistatic agent, pour point depressant, etc. to meet practical requirements. Differences in concentration of the additives highly affect the conductivity $[12,13]$. A possible explanation is that these additives dissociate to form ions and take part in the conduction process. These ions can be caught in potential energy wells formed by large polymer molecules. The distance $b$ could represent here the mean distance between the sites at polymer molecules where an ion can be caught.

2. Molecules with electron affinity can be present in the insulation. When these molecules are in the vicinity of polymer molecules, they can take away an electron [14]. Their presence facilitates electron transfer from polymer to molecule. The transferred electron can be retained by the molecule and move as a stable negative ion until it is transferred back to another polymer molecule. The distance $b$ is explained as under 1 .

3. Electrons, ions and other byproducts are the result of partial discharges inside the insulation. The authors and [17] suggest that they can act as charge carriers and take part in the conduction process as under 1 and 2.

4. The oil in mass impregnated paper is much more conductive than the paper [18]. It follows that the conduction current proceeds mainly in the oil phase along the pores in the impregnated paper. According to [15], the pores of the paper fix a large amount of ions of both signs, with one sign dominating. This results in a net charge on the surface of the pores. Ions of the same sign, which take part in the conduction process, experience an electrostatic repulsion force while traveling through the pores (Figure 4). The repulsion forces can be described by the potential energy wells. The distance $b$ would represent here the mean distance between two centers of electrostatic repulsion forces.

5. Any combination of 1 to 4 can occur, this in agreement with Equation (10).

\section{CONCLUSIONS}

$A$ model based on physical considerations has been proA posed describing ionic conduction in mass impregnated paper. This model takes into account the temperature and stress dependency of the insulation. A comparison has been made with the widely used empirical formula for mass impregnated paper. Computed data from the empirical and the physical based formula show an astonishing similarity over a wide range of temperatures and stresses, reflecting the actual service conditions of HVDC cables. This similarity of data makes it most likely that the main process of conduction in mass impregnated paper is of the ionic kind.

A link between the constants in the empirical formula and the constants in the theoretical formula has been made. Data for the carrier density, the distance between 
equilibrium points and the mean height of potential energy wells have been given. The data apply to mass impregnated paper insulation.

Finally, some possible ionic conduction mechanisms, which are likely to happen in industrial mass impregnated paper, are mentioned.

\section{REFERENCES}

[1] F. H. Buller, "Calculation of Electrical Stresses in dc Cable Insulation", IEEE Summer Power Meeting, New Orleans, La., July 10-15, 1966.

[2] E. Occhini and G. Maschio, "Electrical Characteristics of Oil-impregnated Paper as Insulation for HVDC Cables", IEEE Trans. on PAS, Vol. 86, pp. 312-326, March 1967.

[3] J. M. Oudin, M. Fallou and H. Thévenon, "Design and Development of dc Cables", IEEE Trans. on PAS, Vol. 86, pp. 304-311, March 1967.

[4] S. C. Chu, "Design Stresses and Current Ratings of Impregnated Paper Insulated Cables for HVDC", IEEE on PAS, Vol. 86, pp. 1029-1036, September 1967.

[5] Francis H. Buller, "Calculation of Electrical Stresses in dc Cable Insulation", IEEE on PAS, Vol. 86, pp. 1170-1178, October 1967.

[6] G. Maschio and E. Occhini, "High Voltage Direct Current Cables: the state of the Art", International Conference on Large HV Electric Systems, Group 21-10, August 1974.

[7] B. R. Nyberg, K. Herstad and K. Bjorlow-Larsen, "Numerical Methods for Calculation of Electrical Stresses in HVDC Cables with Special Application to the Skagerrak Cablen, IEEE Trans. on PAS, Vol. 94, pp. 491-497, March/April 1975.

[8] "A 200 km-long Submarine Cable for the Fennoskan HVDC Link", ed. O. Tollerz, ABB review, March 1989.
[9] Ulrich Kull, "Das Verhalten Des Geschichteten Ölpapier-dielektrikums Bei Hoher Gleichspannung", Brown Boveri Mitteilungen, Vol. 55, no. 4/5, pp. 215-221, April/May 1968.

[10] H. Frölich, Theory of Dielectrics, Chapter 3, Oxford at the Clarendon Press 1949.

[11] J. C. Anderson, Dielectrics, Chapter 6, Chapman and Hall Ltd. 1967.

[12] S. Yasufuku, T. Umemura and T. Tanii, "Electric Conduction Phenomena and Carier Mobility Behavior in Dielectric Fluids", IEEE Trans. on Electr. Ins., Vol. 14, pp. 28-35, February 1979.

[13] T. Umemura, K. Akiyama and T. Kawasaki, "Electrical Conduction in Synthetic Insulat Ing Liquid", IEEE Trans. on Electr. Ins., Vol. 17, pp. 533-538, December 1982.

[14] P. Karanja and R. Nath, "Charge Trapping and Conduction in Pure and Iodine-doped Biaxiallyoriented Polypropylene", IEEE Trans. on Diel. and Electr. Ins., Vol. 1, pp. 213-223, April 1994.

[15] B. Gosse, J. P. Gosse and M. Sauviat, "Interactions Between the Solid and the Ions of the Liquid in Impregnated Paper", IEEE Trans. on Electr. Ins., Vol. 15, pp. 104-111, April 1980.

[16] J. O'M. Bockris and A. K. N. Reddy, Modern Electrochemistry, Chapters 7 and 8, Plenum Press, 1973.

[17] L. A. Dissado and J. C. Fothergill, Electrical Degradation and Breakdown in Polymers, Chapter 2, Peter Peregrinus Ltd. 1992.

[18] E. Takahashi, Y. Tsutsumi, K. Okuyama and F. Ogata, "Partial Discharge Characteristics of Oilimmersed Insulation Systems under dc, Combined ac-dc And dc Reversed Polarity Voltage", IEEE Trans. on PAS, Vol. 95, no. 1 Jan/Feb 1976.

[19] J. J. O'Dwyer, The Theory of Electrical Conduction and Breakdown in Solid Dielctrics, Chapters 1 and 4, Clarendon Press, 1973.

Manuscript was received on 2 March 1995, in revised form 5 July 1995. 